\title{
Participación y dinamicidad en las Infraestructuras de Datos Espaciales: una propuesta de indicadores para medir su impacto en la sociedad
}

\author{
Diego Randolf Perez* \\ Daniela Ballari** \\ Luis M. Vilches-Blázquez ${ }^{* * *}$
}

Recibido el 16 de junio de 2015; aceptado el 16 de agosto de 2015

\begin{abstract}
Evaluation and indicators definition is an issue in the context of Spatial Data Infrastructures (SDI). Previous studies have evaluated first and second generations of SDI in comparison to their planned or anticipated development. This is following an exante approach. However, current SDI trends requires consideration of additional elements for a proper evaluation. The rise of mobile devices, sensors and the growing citizen collaboration are driven the SDI development to a third generation which demands interactive contents, frequently updates, real-time and participation. This paper proposes a system of indicators aimed at evaluating the performance of new SDI trends under an ex-post approach, i.e. after its development. The indicator system assesses two dimensions of management processes that occur in the GeoWeb: participation (through dialogue and empowerment) and dynamism. This work contributes to the improvement of existing SDI evaluation systems by integrating specific indicators for new technology trends.

Key words: New technological trends, mobile devices, Sensors, Volunteered Geographic Information, GeoWeb.

* IDECA, Catastro Distrital. Av. Carrera 30 No. 25-90, Bogotá, Colombia, correo electrónico: diego.randolf@gmail.com

** Grupo de Geoinformación, Departamento de Ingeniería Civil, Facultad de Ingeniería, Universidad de Cuenca, Avenida 12 de Abril y Agustín Cueva, Cuenca, Ecuador, correo electrónico: daniela.ballari@ucuenca.edu.ec

*** Facultad de Ingeniería, Pontificia Universidad Javeriana, Calle 40 No. 5-50, Ed. Maldonado, Bogotá, Colombia, correo electrónico: lmvilches@javeriana.edu.co
\end{abstract}




\section{Resumo}

A avaliação e definição de indicadores é o centro das atenções no contexto da comunidade de Infraestruturas de Dados Espaciais (IDE). Trabalhos anteriores têm avaliado as IDE de primeira e segunda geração em comparação com o seu planejamento e desenvolvimento, utilizando uma abordagem ex-ante. No entanto, a evolução atual das IDE requere a consideração de outros elementos para que se chegue uma avaliação adequada. O auge dos dispositivos móveis, os sensores ubíquos e a crescente participação cidadã estão direcionando o desenvolvimento das IDE a uma terceira geração na qual é necessária a interatividade de conteúdos, atualizações frequentes, tempo real e participação.Este artigo propõe um sistema de indicadores com a finalidade de avaliar o desempenho das novas tendências das IDE com um enfoque ex-post, ou seja, após a sua implantação. O sistema de indicadores avalia duas dimensões dos processos de gestão que se dão na GeoWeb: participação (através do diálogo e empoderamento) e dinamicidade. Este trabalho contribui para a melhoria dos atuais sistemas de avaliação de IDE, integrando indicadores específicos para novas tendências tecnológicas.

Palavras chave: novas tendências tecnológicas, dispositivos móveis, sensores, Informação Geográfica Voluntaria, GeoWeb.

\section{Resumen}

La evaluación y definición de indicadores es centro de atención en el contexto de la comunidad de las Infraestructuras de Datos Espaciales (IDE). Trabajos previos han evaluado las IDE de primera y segunda generación en comparación con lo planificado o previsto en su desarrollo, es decir siguiendo un enfoque ex-ante. Sin embargo, la evolución actual de las IDE requiere considerar otros elementos en juego para su adecuada evaluación. El auge de los dispositivos móviles, los sensores ubicuos y la creciente colaboración ciudadana están direccionando el desarrollo de las IDE hacia una tercera generación en la que se demanda interactividad de contenidos, actualización frecuente, tiempo real y participación. Este artículo propone un sistema de indicadores orientado a evaluar el desempeño de las nuevas tendencias IDE bajo un enfoque ex-post, es decir posterior a su desarrollo. El sistema de indicadores evalúa dos dimensiones de los procesos de gestión que se dan en la GeoWeb: participación (a través del diálogo y empoderamiento) y dinamicidad. Este trabajo contribuye a la mejora de los sistemas actuales de evaluación de IDE, integrando indicadores específicos para nuevas tendencias tecnológicas.

Palabras clave: nuevas tendencias tecnológicas, dispositivos móviles, sensores, Información Geográfica Voluntaria, GeoWeb. 


\section{Introducción}

La identificación de mecanismos de evaluación y definición de indicadores es centro de atención en el contexto de la comunidad de las Infraestructuras de Datos Espaciales (IDE). Trabajos previos han evaluado las IDE en comparación con lo planificado o previsto en su desarrollo (Rajabifard, Feeney y Williamson, 2002). Por ejemplo, se midió la eficacia, eficiencia y fiabilidad en la determinación del estado y desarrollo de las IDE (Giff, 2008). En esta misma línea, INSPIRE (Infrastructure for Spatial Information in Europe) propuso 32 indicadores iniciales para medir el estado y desarrollo de las diferentes IDE europeas que la conforman con el objetivo de describir, monitorear y analizar sus actividades (SADL, 2005). Otras iniciativas centraron el interés en el desarrollo de los Clearinghouse IDE (Crompvoets, Bregt, Rajabifard y Williamson, 2004) y en la dimensión económica (Zwirowicz-Rutkowska, 2013).

Todos estos enfoques, sin embargo, fueron construidos para evaluar las IDE de primera y segunda generación. Es decir, estas iniciativas se caracterizaban por estar centradas en los datos, servicios y procesos y fueron impulsadas por instituciones gubernamentales y empresas privadas (Masser, 1999). Por consiguiente, su evaluación está basada en el desempeño de productos (calidad) en términos de eficiencia, efectividad y eficacia de productos y objetivos.

No obstante, la evolución actual de las IDE requiere considerar otros elementos en juego para su adecuada evaluación. Ante el auge de los dispositivos móviles, los sensores ubicuos y la creciente colaboración ciudadana en la construcción de contenidos, las IDE se posicionan frente a entornos complejos, multifacéticos y dinámicos (Erik de Man, 2006; Grus, Crompvoets y Bregt, 2010). Estas tendencias están direccionando el desarrollo de las IDE hacia una tercera generación en la que se demanda interactividad de contenidos, actualización frecuente, tiempo real y participación donde todos los actores sociales puedan proveer, acceder, usar e intercambiar datos espaciales por medio de plataformas colaborativas.

En la actualidad, son diferentes las iniciativas de evaluación de IDE de tercera generación que se han desarrollado (Floreddu y Cabiddu, 2012; Grus et al., 2011; Ho y Rajabifard, 2010). Sin embargo, a pesar de los aportes de estos trabajos y de acuerdo a la revisión realizada, no existen trabajos orientados a medir de forma directa e integral la participación y dinamicidad necesarios en el marco de las nuevas tendencias IDE. Algunos trabajos aportan parámetros aislados de medición (Bégin, Devillers y Roche, 2013; Walker y Claus, 2013), pero carecen de un marco sistemático que permita establecer criterios de evaluación sobre el estado y los retos de implementación de estas tendencias.

Por ello este trabajo desarrolla un sistema de indicadores orientado a evaluar el desempeño de las nuevas tendencias IDE bajo un enfoque ex-post, es decir posterior a su desarrollo. Este sistema de indicadores evalúa dos dimensiones de los procesos 
de gestión que se dan en la GeoWeb: participación (a través del dialogo y empoderamiento) y dinamicidad. Este sistema se basa en el modelo propuesto por Walker y Claus (2013).

El documento se divide en dos secciones. La primera presenta los antecedentes que han determinado el desarrollo y la evaluación de las IDE; la segunda reflexiona sobre los parámetros requeridos para validar las nuevas tendencias y compila cada uno de los indicadores según criterio, característica y técnica evaluativa. Finalmente se aportan conclusiones.

\section{Antecedentes}

\section{Las IDE y sus etapas de desarrollo}

Una IDE se define como la integración de una serie de componentes, entre ellos datos, tecnología, institucionalidad, comunidad, políticas y estándares, que crean una plataforma para que las partes interesadas, tanto usuarias como productoras de datos espaciales, accedan, compartan y utilicen los datos espaciales de forma eficiente y eficaz (Comisión Europea, 2007; Giff y Coleman, 2002; Kok y van Loenen, 2005; Macharis y Crompvoets, 2014).

En el desarrollo de las IDE se reconocen dos etapas o generaciones bien diferenciadas. En la primera generación, las IDE se impulsan para que la información geoespacial producida por las instituciones gubernamentales sea publicada y accedida a través de Internet. Así, el objetivo prioritario de las IDE es desplegar servicios para la visualización de datos espaciales y la búsqueda de metadatos. Por tanto, en esta etapa, las IDE se centran en los datos y son impulsadas por instituciones gubernamentales (Masser, 1999).

La segunda generación comenzó alrededor del año 2000 (Rajabifard, Feeney y Williamson, 2003) y promueve el desarrollo de servicios geoespaciales más especializados, tales como Web Feature Service (WFS), Web Coverage Service (WCS) yWeb Processing Service (WPS). Estos servicios proporcionan funcionalidades adicionales a la simple visualización permitiendo a los usuarios consultar y analizar atributos, acceder a datos en tiempo real y realizar análisis espacial online. En esta etapa, las IDE se centran en los procesos y preferencias de los usuarios.

Actualmente, en pleno siglo XXI y ante el auge de los dispositivos móviles, los sensores ubicuos y la creciente colaboración ciudadana en la construcción de contenidos, las IDE se posicionan frente a entornos complejos, multifacéticos y dinámicos (Erik de Man, 2006; Grus et al., 2010). Estas tendencias están direccionando el desarrollo IDE hacia una tercera generación en la que se demanda interactividad de contenidos, actualización frecuente, tiempo real y participación. Este desarrollo implica pasar de un modelo basado en productos, donde las personas consumen datos (Web 1.0), a un modelo basado en procesos donde todos los actores sociales 
puedan proveer, producir, acceder, usar e intercambiar datos espaciales por medio de plataformas colaborativas ( Web 2.0).

\section{Características de las nuevas tendencias IDE}

La información geográfica voluntaria (VGI en inglés por Volunteer GeoInformation) se caracteriza por ser un compromiso generalizado por parte de un gran número de ciudadanos, a menudo con poca cualificación en términos formales, que participan en la creación de datos espaciales (Low, 2012). Esta es una fuente rica de información casi en tiempo real que supone un cambio de paradigma en la creación y difusión de los datos espaciales. Lo anterior, porque se produce un cambio desde un modelo tradicional, centrado en los productores oficiales, hacia un modelo más democrático donde el usuario final también actúa como productor de datos (Budhathoki, Bruce y Nedovic-Budic, 2008; Goodchild, 2008; Severinsen y Reitsma, 2013).

La VGI se logra gracias a la Web 2.0 y a los mashups, término asociado a aplicaciones web que facilitan el intercambio participativo de información, la interoperabilidad, el diseño centrado en el usuario y la colaboración en la World Wide Web (por ejemplo, los sitios de redes sociales, blogs, wikis, sitios para compartir video, etc.) (Low, 2012). Así, un sitio Web 2.0 se caracteriza por permitir a los usuarios interactuar y colaborar entre sí como creadores de contenido en una comunidad virtual, a diferencia de sitios web estáticos donde los usuarios se limitan a la observación pasiva de los contenidos que se han creado para ellos.

Por su connotación, VGI requiere abordar aspectos relacionados con privacidad, derechos de propiedad intelectual, calidad y responsabilidad de los datos (Pomfret, 2010). El grado de integración de este tipo de información en las IDE implica constituir un sistema de control con el cual se tenga conocimiento tanto por parte del contribuyente como por la administración de: ¿Cómo están siendo adquiridos, administrados, analizados y utilizados los datos? ¿Cuándo y por cuánto tiempo? de forma tal que las personas sean conscientes del tipo de infraestructura a la que contribuyen y los posibles beneficios que pueden obtener de ella (Batty et al., 2012).

Los dispositivos móviles, por otro lado, permiten la navegación táctil de mapas, la interacción con el entorno a través de la disponibilidad de sensores, la movilidad de los usuarios y el reporte de eventos en tiempo real (Goodchild, 2007). De esta forma, dichos instrumentos (smartphones, tablets, etc.) se han incorporado a la vida cotidiana recogiendo, intercambiando y procesando información de forma continua tanto espacial como temporalmente (Cuff, Hansen y Kang, 2008). Esta situación está dando lugar a múltiples datos (localización, trayectorias, velocidades, magnitudes, etc.) de un sinnúmero de objetos y fenómenos geográficos que demandan ser conocidos por la sociedad. 
La generación de datos espaciales también se beneficia de la disponibilidad de sensores que, ya sea de forma independiente o integrada con los dispositivos móviles, monitorean y reportan eventos del medioambiente. Estos sensores ayudan a la observación de fenómenos como dióxido de carbono, luminosidad y ruido (Christin, Reinhardt, Kanhere y Hollick, 2011). Por ello, los geoservicios de las IDE están siendo rediseñados, adaptados y complementados para soportar también los datos de sensores que son dinámicos, accesibles en tiempo real y con una alta resolución espacio-temporal. Esto es llevado adelante por la iniciativa de Sensor Web Enablement (SWE) del Open Geospatial Consortium (OGC) a través de la estandarización de geoservicios específicos para datos de sensores (Bröring et al., 2011).

\section{Indicadores en la evaluación de las IDE}

Los indicadores proporcionan evidencia de la existencia de una cierta condición, es decir del logro o no de ciertos resultados (Brizius y Campbell, 1991). Permiten a los tomadores de decisiones evaluar el progreso hacia el logro de los resultados previstos, metas y objetivos; siendo una parte integral de un sistema de rendición de cuentas basado en los resultados (Horsch, 1997). Constituyen expresiones cualitativas o cuantitativas observables que describen características, comportamientos o fenómenos de la realidad a través de la evolución de una variable o del establecimiento de una relación entre variables (DANE, n.d.). Los indicadores tienen dos funciones básicas. Por un lado, la función descriptiva que consiste en la aportación de información sobre el estado real de una actuación, proyecto, programa o política. Por otro lado, la función valorativa que consiste en añadir a la información descriptiva un juicio de valor, lo más objetivo posible, sobre si el desempeño está siendo o no adecuado (Valle y Rivera, 2008).

Debido a la alta complejidad de las iniciativas IDE, en las que intervienen variadas disciplinas como el derecho, economía, geomática y administración pública (Macharis y Crompvoets, 2014); a la hora de medir el desempeño de estas macroestructuras se identifican múltiples enfoques y criterios de evaluación.

Desde un enfoque general, se desarrolló un marco de trabajo multivista (framework) para evaluar las IDE desde diferentes perspectivas y propósitos (Crompvoets, Rajabifard, van Loenen y Fernández, 2008). Este marco de trabajo se basa en Sistemas Complejos Adaptativos (CAS en sus siglas en inglés) y responde a tres requerimientos básicos de medición: la rendición de cuentas (contabilidad), el conocimiento y el desarrollo de las IDE.

También basándose en el rendimiento de productos (PBM por sus siglas en inglés), se midió la eficacia, eficiencia y fiabilidad en la determinación del estado y desarrollo de las IDE (Giff, 2008). En esta línea, INSPIRE propuso 32 indicadores iniciales para medir el estado y desarrollo de las diferentes IDE europeas con el objetivo de describir, monitorear y analizar sus actividades (SADL, 2005). Otros 
trabajos centraron el interés en el desarrollo de los Clearinghouse IDE (Crompvoets et al., 2004); y en la dimensión económica (Zwirowicz-Rutkowska, 2013).

Estos enfoques, sin embargo, fueron construidos para evaluar las IDE de primera generación y, por consiguiente, su evaluación está basada en el desempeño de productos (calidad) en términos de eficiencia, efectividad y eficacia de productos y objetivos. Además, como resaltan Grus et al. (2011), estos enfoques han tenido, sobre todo, un carácter ex-ante, es decir, centrado en impactos y beneficios predichos de la IDE (M. Craglia et al., 2003; Dufourmont, 2004; Garcia Almirall, Moix Bergadà, Queraltó Ros y Craglia, 2008). Los estudios a posteriori de los beneficios e impactos de IDE siguen siendo escasos (Grus et al., 2011; Lance, Georgiadou y Bregt, 2006) ya que sólo una serie de consideraciones teóricas y bue nas prácticas relacionadas se pueden mencionar (Castelein, Bregt y Pluijmers, 2010; Max Craglia y Campagna, 2010; Genovese, Cotteret, Roche, Caron y Feick, 2009).

En respuesta a esta limitación, recientemente varios autores han propuesto enfoques ex-post. Ejemplos son el marco para analizar y evaluar Public Participation Geographic Information Systems (PPGIS) (Floreddu y Cabiddu, 2012) y la propuesta de 9 parámetros para validar VGI en el contexto de la Web 2.0 (Ho y Rajabifard, 2010). Por otro lado, el grado de cumplimiento de las metas de una IDE ha sido evaluado a través de 4 objetivos : i) la recuperación y uso de información por parte del público y las empresas; ii) el valor económico agregado por las empresas a la información geográfica que el gobierno les suministra; iii) el uso de la información geográfica disponible en los procesos y servicios del gobierno; y iv) la colaboración entre los gobiernos, empresas, universidades e institutos de conocimiento en el desarrollo y mejora de las herramientas clave (Grus et al., 2011). Adicionalmente, se midió el grado en que los datos espaciales fluyen de manera eficiente entre las organizaciones de una IDE, teniendo en cuenta el acceso, intercambio y grado en que los datos son utilizados (Vandenbroucke, Dessers, Crompvoets, Bregt y Van Orshoven, 2013). Este trabajo también consideró el grado en que se satisfacen las demandas en términos de la contribución de los datos a través de: i) la eficiencia y la calidad, ii) la flexibilidad e innovación y iii) la transparencia y fiabilidad de los proceso de trabajo.

\section{Sistema de indicadores para nuevas tendencias IDE}

El desarrollo actual de las IDE, como se constató en la sección anterior, debe gestionarse en un marco amplio de conocimiento compuesto por ciudadanos, empresas, instituciones de gobierno, grupos e individuos que no solo demandan información espacial sino que, según las circunstancias, pueden constituir nodos de producción, organización, recolección e interpretación de datos espaciales para satisfacer múltiples necesidades y tomar decisiones. Con lo cual es preciso descentralizar y democratizar los datos, así como los procesos geoespaciales que los generan y mantienen. 
Tomando como base el trabajo de Walker y Claus (2013), la participación en la GeoWeb se da a través de tres etapas:

I. Diálogo de necesidades de información entre la ciudadanía y el coordinador IDE

II. Empoderamiento a la ciudadanía con herramientas, información y facilitación en atención a diversas formas de participación (análisis, deliberación, argumentación y colaboración)

III. Utilización de contenidos en procesos de toma de decisión

Estas etapas ocurren a través de tres espacios conceptuales: ámbito proveedor (coordinador IDE), ámbito público (territorio) y espacio de interacción (GeoWeb) (véase Figura 1).

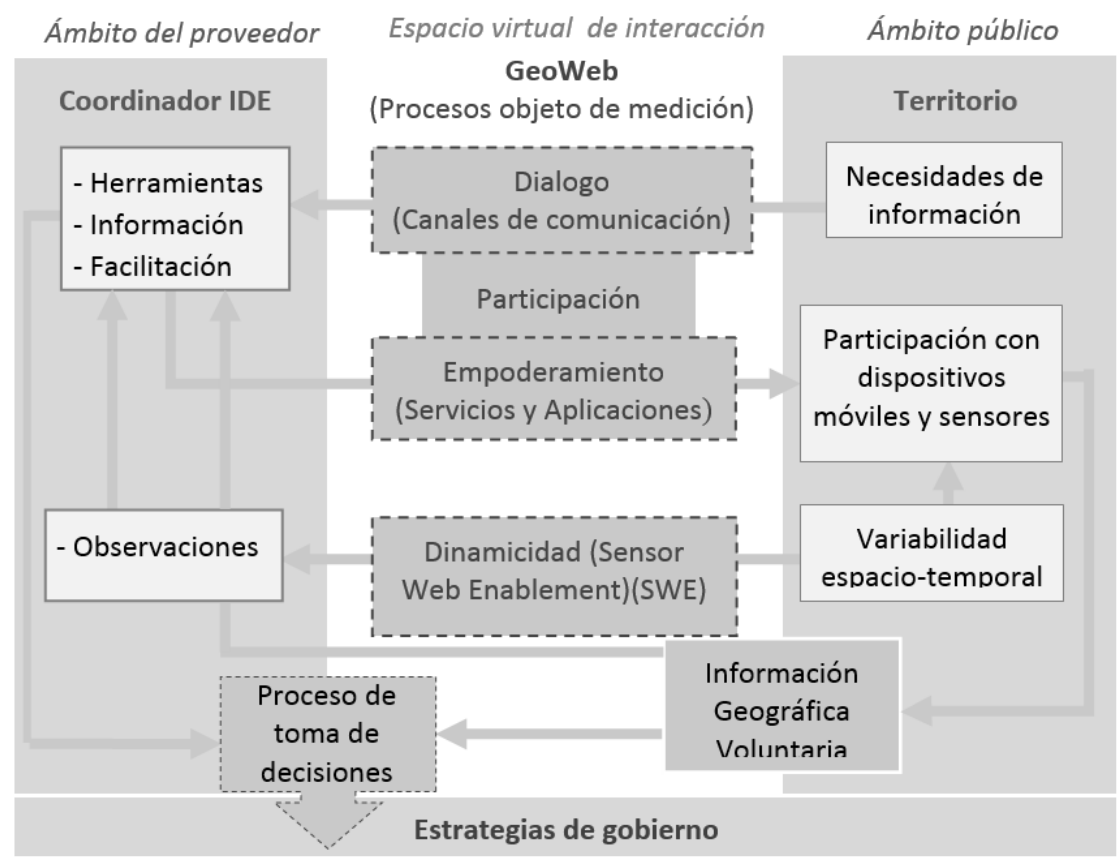

Figura 1. Marco evaluativo para las nuevas tendencias IDE. Basado en (Walker y Claus, 2013).

Este es el escenario que se tomó como elemento de referencia para nuestra propuesta de indicadores para nuevas tendencias IDE (Tablas 1 y 2). El mismo se relaciona con dos procesos principales de la GeoWeb: participación (diálogo y empoderamiento) y dinamicidad, entendiendo estos dos procesos principales como 
formas de interacción entre el proveedor (coordinador IDE) y el ámbito público (territorio).

La Figura 1 muestra como la participación se activa cuando las necesidades de información, que emergen en el territorio, son comunicadas al coordinador IDEa través del diálogo directo y los canales de comunicación. El coordinador IDE, a su vez, genera y dispone herramientas, información y canales de facilitación en atención a estas necesidades; las cuales son puestas al servicio de la ciudadanía como mecanismo de empoderamiento (servicios y aplicaciones) para posibilitar la participación ciudadana que en gran medida se da a través de dispositivos móviles dotados de sensores. Paralelamente, la variabilidad espacio-temporal (dinamicidad) de diversos fenómenos territoriales, es observada por múltiples sensores habilitados en web (Sensor Web Enablement) que son gestionadas por el coordinador IDE. Finalmente y una vez empoderada la ciudadanía, se captura información de forma participativa (información geográfica voluntaria) que ingresa y es utilizada en los procesos de toma de decisión, junto con las herramientas, informaciones y canales facilitados por la IDE; aportando así a la gobernanza y en respuesta a estrategias de gobierno.

Con este escenario para el sistema de indicadores de evaluación se busca visibilizar y dar seguimiento a la información, las herramientas y observaciones que las IDE generan en respuesta a las necesidades de información, formas de participación y variaciones espacio-temporales del territorio, usando estos elementos como parámetros de medida para validar la efectividad y el grado de implementación de las nuevas tendencias IDE.

\section{Participación en la IDE (diálogo y empoderamiento)}

La participación, liderada por los procesos de diálogo y empoderamiento, está regida por la interactividad Web 2.0 definida por Fumero, Roca y Orange (2007) como la interacción entre los distintos elementos de la red universal digital, generalizada en el nuevo entorno tecnológico: hombre-hombre, hombre-máquina, máquinamáquina. Así, la interactividad hombre-máquina habitual de la Web 1.0, se ve transcendida en la Web 2.0 al empujar la "socialización" a una red que pasa a ser una extensión natural del ecosistema social de los individuos (Fumero et al., 2007).

La interactividad de los procesos de diálogo y empoderamiento es una dimensión evaluativa para las IDE que se justifica por la necesidad de integrar procesos de trabajo en los que los datos fluyen entre y dentro de las organizaciones por medio de múltiples mecanismos de intercambio (Vandenbroucke, Crompvoets, Vancauwenberghe, Dessers y Van Orshoven, 2009). Además, estos procesos resultan importantes en la construcción de un recurso de conocimiento global que, por diseño, represente un bien público, accesible a cada ciudadano, institución o empresa dentro de un marco de confianza y calidad que permita garantizar la creación de información confiable, oportuna y fiable sobre los fenómenos colectivos (Batty et al., 2012). 
Tabla 1

Indicadores de diálogo y empoderamiento en la GeoWeb

\begin{tabular}{|c|c|c|}
\hline Indicador & Descripción & $\begin{array}{l}\text { Fuente de } \\
\text { datos }\end{array}$ \\
\hline $\begin{array}{l}\text { Porcentaje de } \\
\text { actividad voluntaria }\end{array}$ & \# total de accesos / \# total de aportes x 100 & \multirow{9}{*}{$\begin{array}{l}\text { Servicios } \\
\text { de análisis } \\
\text { estadístico } \\
\text { para } \\
\text { páginas } \\
\text { web (por } \\
\text { ejemplo: } \\
\text { Google } \\
\text { Analitics) }\end{array}$} \\
\hline $\begin{array}{l}\text { Segmento de } \\
\text { población } \\
\text { participante }\end{array}$ & $\begin{array}{l}\text { Tipo de población que aporta contenidos } \\
\text { (institucional, académico, ciudadano)/ } \\
\text { población total de interés x } 100\end{array}$ & \\
\hline $\begin{array}{l}\text { Amplitud del } \\
\text { grupo de usuarios }\end{array}$ & $\begin{array}{l}\text { Toda la población de interés (total), más del 50\% } \\
\text { de la población (media), menos del } 50 \% \text { de la } \\
\text { población (baja) (adaptado de Vandenbroucke } \\
\text { et al } 2013 \text { ) }\end{array}$ & \\
\hline $\begin{array}{l}\text { Alcance de } \\
\text { intercambio }\end{array}$ & $\begin{array}{l}\text { Todas las partes interesadas (alto), otras } \\
\text { autoridades públicas (medio), organizaciones } \\
\text { activas en el proceso (bajo) (adaptado de } \\
\text { Vandenbroucke } \text { et al., 2013) }\end{array}$ & \\
\hline $\begin{array}{l}\text { Frecuencia de } \\
\text { contribución }\end{array}$ & $\begin{array}{l}\text { Grado en que se aportan datos espaciales al } \\
\text { proceso. Horario, diario, semanal, mensual }\end{array}$ & \\
\hline Intensidad de uso & $\begin{array}{l}\text { Grado en que se utilizan los datos espaciales en el } \\
\text { proceso. Diario (alto), semanal (medio), de vez en } \\
\text { cuando (bajo) (adaptado de Vandenbroucke et al., } \\
2013 \text { ) }\end{array}$ & \\
\hline Ámbito de uso & $\begin{array}{l}\text { Avanzado (alto), analítico (medio), básico (bajo) } \\
\text { (adaptado de Vandenbroucke et al., 2013) }\end{array}$ & \\
\hline $\begin{array}{l}\text { Medio de } \\
\text { interacción } \\
\text { predilecto }\end{array}$ & \# de accesos web / \# de accesos móviles & \\
\hline $\begin{array}{l}\text { Sistema operativo } \\
\text { predilecto }\end{array}$ & \# de accesos por sistemas operativo & \\
\hline $\begin{array}{l}\text { Contenido de } \\
\text { intercambio }\end{array}$ & $\begin{array}{l}\text { Tipo de contenido (especializado, intuitivo)/ Tipo } \\
\text { de contenido requerido. Conforme, no conforme } \\
\text { (adaptado de Vandenbroucke et al., 2013) }\end{array}$ & \multirow{7}{*}{$\begin{array}{l}\text { Capas } \\
\text { espaciales, } \\
\text { sistema de } \\
\text { registro y } \\
\text { formas de } \\
\text { publicación } \\
\text { de un } \\
\text { servicio o } \\
\text { aplicación }\end{array}$} \\
\hline $\begin{array}{l}\text { Grado de } \\
\text { coherencia }\end{array}$ & $\begin{array}{l}\text { \# de aportes inmersos en la zona de interés/ el total } \\
\text { de aportes }\end{array}$ & \\
\hline $\begin{array}{l}\text { geográfica en los } \\
\text { aportes }\end{array}$ & & \\
\hline $\begin{array}{l}\text { Privacidad de los } \\
\text { datos que ingresan }\end{array}$ & $\begin{array}{l}\text { Existencia de licencias (Contiene, No contiene); } \\
\text { Existencia de pseudónimos (Contiene, No } \\
\text { contiene) }\end{array}$ & \\
\hline $\begin{array}{l}\text { Calidad de los aportes } \\
\text { voluntarios }\end{array}$ & $\begin{array}{l}\text { Sistemas de calificación por pares ranking (bueno, } \\
\text { regular o malo) }\end{array}$ & \\
\hline $\begin{array}{l}\text { Difusión de } \\
\text { contenidos }\end{array}$ & $\begin{array}{l}\text { Sindicación web (posee, no posee); conexión con } \\
\text { redes sociales (posee, no posee); publicación por } \\
\text { servicios (posee, no posee) }\end{array}$ & \\
\hline $\begin{array}{l}\text { Porcentaje de } \\
\text { información } \\
\text { voluntaria }\end{array}$ & $\begin{array}{l}\text { \# de dataset voluntarios / } \\
\text { \# de dataset oficiales } \times 100\end{array}$ & \\
\hline
\end{tabular}

Fuente: Elaboración propia. 
De esta manera, los procesos de diálogo y el empoderamiento demandan un esfuerzo de diseño para planificar una navegación entre pantallas en la que el usuario sienta que realmente controla y maneja una aplicación. Es decir, el usuario debe navegar por la aplicación y sentirse libre (Bauzá, 1997) para dialogar con el proveedor y para usar las aplicaciones y servicios que el coordinador IDE otorga para participar. Esto es, un "diseño centrado en el usuario" que tenga en cuenta: i) el intercambio, como flujo y acceso eficiente a datos espaciales entre los diferentes grupos de interés (Annoni y Craglia, 2005); ii) la colaboración, entendida como proceso que integra a las personas y la tecnología bajo el propósito de gestionar, transformar y analizar datos espaciales, permitiendo la integración de conocimientos de múltiples partes interesadas (Balram y Dragićević, 2006) y iii) la interoperabilidad como aquella habilidad de las organizaciones y sistemas dispares y diversos para interaccionar con objetivos consensuados y comunes con la finalidad de obtener beneficios mutuos (Marco Iberoamericano de Interoperabilidad, 2010).

Teniendo en cuenta estos aspectos se proponen los siguientes indicadores para evaluar la interactividad de la participación (diálogo y el empoderamiento).

\section{Dinamicidad en las IDE}

Para poder dimensionar una red de sensores que resulte genérica y adaptable a cualquier escenario geográfico, es preciso tener en cuenta las entidades involucradas en un ejercicio de observación y la forma como estas interactúan o colaboran, pues al armonizar la forma en que cada elemento colabora al momento de integrarse, es posible equilibrar las metas individuales y colectivas (Collazos et al., 2009).

El reconocimiento de la variabilidad espacio-temporal del territorio, a través de sensores conectados y habilitados en Web (esto bajo los protocolo y estándares de Sensor Web Enablement de OGC y la Semantic Sensor Network Ontology (SSN) del World Wide Web (W3C)), es una manera de dotar de órganos sensoriales a las IDE que permite a los usuarios explorar el entorno (Rodríguez Pascual, López Romero, Abad Power, Sánchez Maganto y Vilches-Blázquez, 2005).

De acuerdo con la SSN, un sensor puede definirse como toda cosa que observa y permite describir cualquier nivel de detalle. De esta manera, los sensores son objetos físicos que observan, que transforman los estímulos entrantes en otra representación, a menudo digital, a través de un método.

En términos concretos, la red de sensores y dispositivos móviles se puede evaluar de acuerdo a tres perspectivas (Compton et al., 2012): i) la capacidad de medición de los sensores, (exactitud, rango de medición, precisión, resolución y similares); ii) observaciones, contextos de interpretación de los estímulos de entrada que colocan el estímulo y el caso observado en un contexto de interpretación y iii) el sistema de detección y despliegue, partes de detección de la infraestructura 
(componentes, dispositivos de detección, funcionamiento, supervivencia, plataformas y ciclo de despliegue: instalación, mantenimiento y desmantelamiento).

Para un evento una observación puede vincular el acto de percibir, el evento de estímulo, el sensor, un método, un resultado, una característica y una propiedad observada, colocando todo en un contexto de interpretación (Compton et al., 2012). Partiendo de este entendimiento se proponen los indicadores recogidos de la Tabla 2.

Tabla 2

\section{Indicadores de dinamicidad en la GeoWeb}

\begin{tabular}{|c|c|c|}
\hline Indicador & Descripción & $\begin{array}{l}\text { Fuente } \\
\text { de datos }\end{array}$ \\
\hline $\begin{array}{l}\text { Frecuencia de } \\
\text { aportes }\end{array}$ & \#aportes u observaciones/unidad de tiempo & \\
\hline $\begin{array}{l}\text { Nivel de } \\
\text { disponibilidad entre } \\
\text { datos } \\
\text { móviles y web }\end{array}$ & $\begin{array}{l}\text { \# Contenidos disponibles en web/\#contenidos } \\
\text { disponibles móvil }\end{array}$ & $\begin{array}{l}\text { Conjunto de } \\
\text { especificaciones }\end{array}$ \\
\hline $\begin{array}{l}\text { Calidad del } \\
\text { instrumento } \\
\text { sensor }\end{array}$ & $\begin{array}{l}\text { Exactitud del instrumento/exactitud } \\
\text { requerida }\end{array}$ & $\begin{array}{l}\text { del Sensor Web } \\
\text { Enablement } \\
\text { (SWE). } \\
\text { Servicios de }\end{array}$ \\
\hline $\begin{array}{l}\text { Grado de } \\
\text { actualización }\end{array}$ & $\begin{array}{l}\text { Periodicidad de captura/periodicidad de } \\
\text { publicación }\end{array}$ & $\begin{array}{l}\text { análisis } \\
\text { estadístico para } \\
\text { páginas web }\end{array}$ \\
\hline $\begin{array}{l}\text { Grado de análisis de } \\
\text { información }\end{array}$ & $\begin{array}{l}\text { Publicación de datos crudos/publicación de datos } \\
\text { procesados x } 100\end{array}$ & $\begin{array}{l}\text { (por ejemplo } \\
\text { Google } \\
\text { Analitics) }\end{array}$ \\
\hline $\begin{array}{l}\text { Disponibilidad de } \\
\text { datos de trayectos }\end{array}$ & \# de dataset que registren trayectorias & \\
\hline $\begin{array}{l}\text { Representatividad } \\
\text { temporal del evento }\end{array}$ & $\begin{array}{l}\text { Periodo de actividad del sensor/tiempo de } \\
\text { ocurrencia del fenómeno }\end{array}$ & \\
\hline
\end{tabular}

Fuente: Elaboración propia.

\section{Conclusiones}

Este trabajo ha desarrollado un sistema de indicadores orientado a evaluar el desempeño de las nuevas tendencias IDE bajo un enfoque ex-post, es decir, que se centra en analizar el impacto social de las nuevas tendencias IDE como son la participación ciudadana y la dinamicidad de información. Esta propuesta resulta dife- 
renciadora con respecto a los trabajos presentes en el estado del arte, caracterizados por seguir enfoques ex-antes, es decir, enfoques centrados en impactos y beneficios predichos de las iniciativas IDE. Los indicadores propuestos fueron obtenidos de referencias bibliográficas y mediante discusión entre los autores. También se indican fuentes de datos para así poder realizar la evaluación de los mismos.

Los indicadores de evaluación ex-post propuestos permiten evaluar la IDE atendiendo los procesos de comunicación y empoderamiento tecnológico que el coordinador IDE establece con la ciudadanía a través de la GeoWeb. De esta manera, la evaluación del desempeño, se centra en el impacto que las herramientas, informaciones y facilidades IDE imparten sobre el territorio. Es decir en el grado de atención a las necesidades de información y en el retorno de mecanismos de participación y monitoreo web y móvil que la IDE dispone para observar la variabilidad espacio-temporal (dinamismo) y gracias a los cuales la ciudadanía se empodera para producir Información Geográfica Voluntaria.

Este trabajo contribuye a la mejora de los sistemas actuales de evaluación de IDE, integrando indicadores específicos para nuevas tendencias tecnológicas. Como trabajo futuro se platea llevar a cabo diversos casos de estudios que permitan comprobar la factibilidad de los indicadores propuestos y enriquecer la propuesta desarrollada añadiendo una tercera dimensión para evaluar el uso de la información en los procesos de toma de decisiones.

\section{Agradecimientos}

Este trabajo fue llevado a cabo en el marco del proyecto "Escenarios para el análisis de las nuevas tendencias en IDE en Latinoamérica: retos y oportunidades" financiado por el Instituto Panamericano de Geografía e Historia (IPGH).

\section{Bibliografía}

Annoni, A., and Craglia, M. (2005). Towards a directive establishing an infrastructure for spatial information in Europe (INSPIRE) in Proceedings of GSDI -8 From Pharaohs to Geoinformatics-The Role of SDIs in an Information Society, Cairo, April.

Balram, S. and Dragićević, S. (2006). Collaborative Geographic Information Systems, Idea Group Inc (IGI).

Batty, M.; Axhausen, K.W.; Giannotti, F.; Pozdnoukhov, A.; Bazzani, A.; Wachowicz, M.; Portugali, Y. (2012). "Smart cities of the future", The European Physical Journal Special Topics, 214(1), 481-518.

Bauzá, G.B. (1997). El guión multimedia, Universidad Autònoma de Barcelona.

Bégin, D.; Devillers, R. and Roche, S. (2013). "Assessing volunteered geographic information (VGI) quality based on contributors'mapping behaviours", ISPRS- 
International Archives of the Photogrammetry, Remote Sensing and Spatial Information Sciences, 1(1), 149-154.

Brizius, J.A. and Campbell, M.D. (1991). Getting Results: A Guide for Government Accountabiblity. Council of Governors Policy Adivisors.

Bröring, A.; Echterhoff, J.; Jirka, S.; Simonis, I.; Everding, T.; Stasch, C. and Lemmens, R. (2011). "New generation sensor web enablement", Sensors, 11(3), 2652-2699.

Budhathoki, N.R.; Bruce, B. and Nedovic-Budic, Z. (2008). "Reconceptualizing the role of the user of spatial data infrastructure. GeoJournal", 72(3-4), 149-160.

Castelein, W., Bregt, A. and Pluijmers, Y. (2010). "The economic value of the Dutch geo-information sector", International Journal of Spatial Data Infraestructures Research, 5, 58-76.

Christin, D.; Reinhardt, A.; Kanhere, S.S. and Hollick, M. (2011). "A survey on privacy in mobile participatory sensing applications", Journal of Systems and Software, 84(11), 1928-1946.

Collazos, C.A.; Alvira, J.A.; Martínez, D.F.; Jiménez, J.; Cobos, R. y Moreno, J. (2009). "Evaluando y monitoreando actividades colaborativas en dispositivos móviles", Avances en sistemas e informática, 5(1).

Comisión Europea. (2007). Directive 2007/2/EC of the European Parliament and of the Council of 14 March 2007: Establishing an Infrastructure for Spatial Information in the European Community (INSPIRE).

Compton, M.; Barnaghi, P.; Bermudez, L.; García-Castro, R.; Corcho, O.; Cox, S., et al. (2012). The SSN ontology of the W3C semantic sensor network incubator group. Web Semantics: Science, Services and Agents on the World Wide Web, $17,25-32$.

Craglia, M. and Campagna, M. (2010). "Advanced regional SDIs in Europe: Comparative cost-benefit evaluation and impact assessment perspectives", International Journal of Spatial Data Infrastructures Research, 5, 145-167.

Craglia, M., et al. (2003). Contribution towards the extended impact assessment of INSPIRE. Environment Agency for England and Wales. Retrieved from $<\mathrm{http} / / /$ inspire.ec.europa.eu/reports/fds_report.pdf $>$.

Crompvoets, J., Bregt, A., Rajabifard, A. and Williamson, I. (2004). "Assessing the worldwide developments of national spatial data clearinghouses", International Journal of Geographical Information Science, 18(7), 665-689.

Crompvoets, J., Rajabifard, A., van Loenen, B., and Fernández, T. (Eds.). (2008). A multi-view framework to assess SDIs. Wageningen: Wageningen University, RGI.

Cuff, D.; Hansen, M. and Kang, J. (2008). "Urban sensing: out of the woods", Communications of the ACM, 51(3), 24-33. 
DANE - Departamento Administrativo Nacional de Estadística (n.d.). Guía para diseño, construcción e interpretación de indicadores.

Dufourmont, H. (2004). Results task force XIA. Luxembourg: EUROSTAT. Retrieved from $<$ http://inspire.jrc.ec.europa.eu/reports/inspire_extended impact_assessment.pdf $>$.

Erik de Man, W.H. (2006). "Understanding SDI; complexity and institutionalization”, International Journal of Geographical Information Science, 20(3), 329-343, <http://doi.org/>.

Floreddu, P.B., and Cabiddu, F. (2012). "Public Decisions and Citizen Satisfaction: The Potential Role of Public Participation Geographic Information Systems". International Journal of Electronic Commerce, 3(1).

Fumero, A.; Roca, G. and Orange, F. (2007). Web 2.0. Fundación Orange.

Garcia Almirall, P.; Moix Bergadà, M.; Queraltó Ros, P. and Craglia, M. (2008). "The socio-economic impact of the spatial data infrastructure of Catalonia", JRC Scientific and Technical Reports.

Genovese, E.; Cotteret, G.; Roche, S.; Caron, C. and Feick, R. (2009). "Evaluating the socio-economic impact of Geographic Information: A classification of the literature", International Journal of Spatial Data Infrastructures Research, 4, 218-238.

Giff, G. (2008). “A Framework for Designing Performance Indicators for Spatial Data Infrastructure Assessment", in A Multi-view framework to assess spatial data infrastructures (in J. Crom, pp. 211-234), Melbourne, Melbourne University Press.

Giff, G. and Coleman, D. (2002). "Funding models for SDI implementation: From local to global", in Proceedings of GSDI6 conference on SDI.

Goodchild, M.F. (2007). Editorial: Citizens as Voluntary Sensors: Spatial Data Infrastructure in the World of Web 2 . 0. International Journal, 2(2), 24-32. $<$ http://doi.org/10.1016/j.jenvrad.2011.12.005>.

Goodchild, M.F. (2008). “Commentary: whither VGI?”, GeoJournal, 72(3-4), 239244.

Grus, L.; Castelein, W.; Crompvoets, J.; Overduin, T.; Loenen, B. van; Groenestijn, A. van, ... Bregt, A.K. (2011). An assessment view to evaluate whether Spatial Data Infrastructures meet their goals. Computers, Environment and Urban Systems, 35(3), 217-229. <http://doi.org/10.1016/j.compenvurbsys.2010.09. $004>$.

Grus, L.; Crompvoets, J. and Bregt, A.K. (2010). "Spatial data infrastructures as complex adaptive systems", International Journal of Geographical Information Science, 24(3), 439-463, <http://doi.org/10.1080/13658810802687319>.

Ho, S. and Rajabifard, A. (2010). Learning from the crowd: The role of volunteered geographic information in realising a spatially enabled society. In Proceedings 
of the 12th GSDI World Conference, Realising Spatially Enabled Societies, Singapore, pp. 19-22.

Horsch, K. (1997). Indicators: Definition and Use in a Results-Based Accountability System. Cambridge: Harvard Family Research Project.

Kok, B. and van Loenen, B. (2005). "How to assess the success of National Spatial Data Infrastructures?", Computers, Environment and Urban Systems, 29(6), 699-717, <http://doi.org/10.1016/j.compenvurbsys.2004.02.001>.

Lance, K.T.; Georgiadou, Y. and Bregt, A. (2006). "Understanding how and why practitioners evaluate SDI performance", International Journal of Spatial Data Infrastructure Research, 1, 65-104.

Low, H.A. (2012). Volunteered Geographic Information (VGI) Primer, Canadian Geospatial Data Infrastructure. Retrieved from <http://wmsmir. cits.rncan.gc.ca/index.html/pub/geott/ess_pubs/291/291948/cgdi_ip_21e.pdf $>$.

Macharis, C. and Crompvoets, J. (2014). A stakeholder-based assessment framework applied to evaluate development scenarios for the spatial data infrastructure for Flanders. Computers, Environment and Urban Systems. $<$ http://doi.org/10.1016/j.compenvurbsys.2014.04.001>.

Marco Iberoamericano de Interoperabilidad. (2010). Bases para una Estrategia Iberoamericana de Interoperabilidad. Documento marco iberoamericano de interoperabilidad ratificado en 2010 por la XX Cumbre Iberoamericana de Jefes de Estado y de Gobierno.

Masser, I. (1999). "All shapes and sizes: the first generation of national spatial data infrastructures", International Journal of Geographical Information Science, $13(1), 67-84$.

Pomfret, K. (2010). "Spatial Law and Policy". Legal and policy issues associated with geospatial data and technology [blog].

Rajabifard, A.; Feeney, M.-E.F. y Williamson, I. (2003). Spatial data infrastructures: concept, nature and SDI hierarchy. In Developing Spatial Data Infrastructures: From Concept to Reality (pp. 17-37). CRC Press, Boca Raton.

Rajabifard, A.; Feeney, M.-E.F. and Williamson, I.P. (2002). "Future directions for SDI development", International Journal of Applied Earth Observation and Geoinformation, 4(1), 11-22, <http://doi.org/10.1016/S0303-2434(02)00002-8>. Rodríguez Pascual, A.F.; López Romero, E.; Abad Power, P.; Sánchez Maganto, A. and Vilches-Blázquez, L.M. (2005). Nuevos roles en el nuevo paradigma IDE. Retrieved from $<$ http://oa.upm.es/7388/1/Nuevos.pdf $>$.

SADL. (2005). Spatial Data Infrastructure in Europe: state of play during 2005.

Severinsen, J. and Reitsma, F. (2013). Finding the Quality in Quantity: Establishing Trust For Volunteered Geographic Information.

Valle, O. and Rivera, O. (2008). Monitoreo e indicadores. IDIE Guatemala. Educación inicial y Derechos de la infancia, Sevilla, Junta de Andalucía. 
Vandenbroucke, D.; Crompvoets, J.; Vancauwenberghe, G.; Dessers, E. and Van Orshoven, J. (2009). A Network Perspective on Spatial Data Infrastructures: Application to the Sub-national SDI of Flanders (Belgium). Transactions in GIS, 13(s1), 105-122.

Vandenbroucke, D.; Dessers, E.; Crompvoets, J.; Bregt, A.K., and Van Orshoven, J. (2013). "A methodology to assess the performance of spatial data infrastructures in the context of work processes", Computers, Environment and Urban Systems, 38, 58-66, <http://doi.org/10.1016/j.compenvurbsys.2012.12.001>.

Walker, B. and Claus, R. (2013). "A Qualitative Framework for Evaluating Participation on the Geoweb", Journal of the Urban and Regional Information Systems Association, 25(2), 15-24.

Zwirowicz-Rutkowska, A. (2013). Economic dimension of spatial data infrastructure - Overview of assessment approaches and methods, 1, 709-715, <http://doi.org/10.5593/SGEM2013/BB2.V1/S11.013>. 